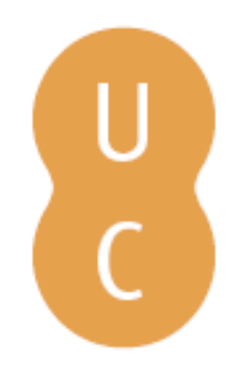

\title{
nombalina
}

\section{Condominio Mitologico di Patrizia Monaco: una casa del Duemila con vista sul passato}

\author{
Autor(es): $\quad$ Trovato, Roberto \\ Publicado por: Imprensa da Universidade de Coimbra; Annablume \\ URL \\ persistente: URI:http://hdl.handle.net/10316.2/40932 \\ DOI: $\quad$ DOI:https://doi.org/10.14195/978-989-26-1298-0_26 \\ Accessed : $\quad$ 26-Apr-2023 16:36:20
}

A navegação consulta e descarregamento dos títulos inseridos nas Bibliotecas Digitais UC Digitalis, UC Pombalina e UC Impactum, pressupõem a aceitação plena e sem reservas dos Termos e Condições de Uso destas Bibliotecas Digitais, disponíveis em https://digitalis.uc.pt/pt-pt/termos.

Conforme exposto nos referidos Termos e Condições de Uso, o descarregamento de títulos de acesso restrito requer uma licença válida de autorização devendo o utilizador aceder ao(s) documento(s) a partir de um endereço de IP da instituição detentora da supramencionada licença.

Ao utilizador é apenas permitido o descarregamento para uso pessoal, pelo que o emprego do(s) título(s) descarregado(s) para outro fim, designadamente comercial, carece de autorização do respetivo autor ou editor da obra.

Na medida em que todas as obras da UC Digitalis se encontram protegidas pelo Código do Direito de Autor e Direitos Conexos e demais legislação aplicável, toda a cópia, parcial ou total, deste documento, nos casos em que é legalmente admitida, deverá conter ou fazer-se acompanhar por este aviso.

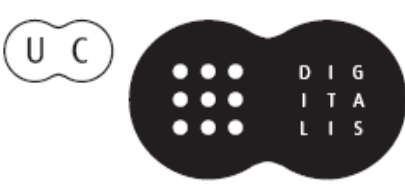




\section{O Livro do Tempo: \\ Escritas e reescritas}

\section{Teatro Greco-Latino e sua recepção II}

Maria de Fátima Silva, Maria do Céu

Fialho \& José Luís Brandão (coords.)

IMPRENSA DA UNIVERSIDADE DE COIMBRA 


\title{
Condominio Mitologico di Patrizia Monaco: \\ Una CASA Del Duemila con Vista sul Passato \\ (Patrizia Monaco's Condominio mitologico: a house of 2000 with a view to the past)
}

\author{
Roberto Trovato (roberto.trovato5@tin.it) \\ Università di Genova, Facoltà di Lettere e Filosofia
}

\begin{abstract}
Riassunto - Condominio mitologico della Monaco è una pièce che parla in maniera originale ed intelligente di temi e personaggi ispirati al mondo della mitologia. Il testo si misura con efficacia col genere socio-politico grazie ad una scrittura compatta e all'abile costruzione modulare.
\end{abstract}

Parole chiave - Studio, testo teatrale, temi e personaggi mitologici, drammaturgia contemporanea, Italia.

Abstract - Condominio mitologico di Patrizia Monaco is a play that talks, in an original and intelligent manner, about themes and characters inspired by the mythology world. The text is measured effectively with the socio-political genre thanks to a compact writing and a skilled modular construction.

Keywords - Analysis, play, mythological themes and characters, contemporary playwriting, Italy.

L'analisi di questo testo di Patrizia Monaco, commediografa vincitrice tra l'altro di prestigiosi premi teatrali, quali il Riccione, l'Idi, il Vallecorsi, il Fersen, il Donne e Teatro, l'Anticoli Corrado, e che da molti anni scrive e traduce per il teatro e per la radio non solo in Italia, ma anche all'estero, si rivela interessante tra le altre ragioni per la sorprendente adattabilità dell'autrice alle richieste del mercato, senza peraltro venire mai meno alla coerenza dell'impegno e dei principi etici che stanno alla base del suo rigoroso percorso artistico, e per la tempestività con cui essa si confronta con eventi che accadono attorno a noi. Inoltre la pièce di questa drammaturga, di cui mi sono occupato altre volte ${ }^{1}$, rivela una scrittrice capace di rileggere criticamente e in maniera originale temi e personaggi ispirati al mondo della mitologia, come farà sette anni dopo con Penelopeide ${ }^{2}$, lavoro scandagliato con grande intelligenza da una studiosa di valore come Milagro Martin Clavijo. Di Condominio mitologico che mi accingo ad analizzare partendo dalla sua genesi e per arrivare alle considerazioni sulla sua struttura definitiva, si

${ }^{1}$ Cf. Trovato 2002: 188-190; Trovato 2012: 1321-1345; Trovato 2014: 1-13 e Trovato 2016: 9-28.

${ }^{2}$ Di questo testo parlo nella già citata introduzione a Monaco 2016: 22-27. 
era peraltro interessata in poche e acute notazioni la sopra ricordata saggista spagnola ${ }^{3}$.

La parte iniziale della pièce nasce all'indomani dell'11 settembre 2001 dall'invito fatto all'autrice dalla Compagnia il Carro dell'Orsa, su sollecitazione della regista, ora scomparsa, Maddalena Falucchi, e di Maria Alessandra Giuri, che le chiesero di scrivere un monologo per un solo attore su una divinità pagana da recitare nell'ambito della manifestazione Lo specchio di Narciso, la figura maschile fra mito e contemporaneità, patrocinata dal Comune di Roma e dall'Ambasciata greca. L'evento venne realizzato con buon successo di pubblico e di critica al Cafè Notegen, di Via del Babuino di Roma, nel novembre di quell'anno. Il componimento, intitolato Ares, la penultima verità, era un breve testo per voce maschile, in cui veniva condannata la guerra senza eccezioni. Anche in questa originaria stesura il copione, preceduto da un prologo scritto nell'imminenza dello spettacolo, che peraltro venne tolto alla vigilia del debutto per non appesantire la rappresentazione, comprova il rilievo che la classicità ha in questa autrice. In effetti per la Monaco, donna di vaste letture, il mondo classico, come preciserò più avanti, è il simbolo di quella patria più profonda e umana alla quale guarda ancora la nostra cultura. L'autrice in un colloquio recente mi ha così spiegato le ragioni della scelta di Ares quale protagonista della pièce:

Dopo una ricerca sui testi di mitologia e saggi sui miti, mi imbattei in un libro di Joseph Campbell, Il potere del mito, che mi intrigò per quanto scritto nella quarta di copertina: 'La mitologia non è una bugia, la mitologia è poesia $\mathrm{e}$ metafora. Si dice che la mitologia sia la penultima verità perché l'ultima non può essere tradotta in parole'.

La lettura di quel volume dell'autorevole esponente statunitense della mitologia comparatista (1904-1987) aiutò l'autrice, "pacifista fino al midollo, avendo passato la giovinezza nelle marce contro la guerra nel Vietnam", scegliere come protagonista del lavoro il dio della guerra. In un'altra occasione la Monaco mi ha dichiarato:

Mi seduceva anche un'idea piuttosto kitsch e nazional-popolare, il pianeta Marte governa il mio segno zodiacale, Ariete, e mi divertiva l'idea cosmica di essere in qualche modo imparentata proprio col dio della guerra, e quindi, quale occasione migliore per conoscerlo meglio? E che ghiotta occasione poter fare parlare della guerra non le vittime, questo lo sanno fare tutti, bensì i carnefici. Salvo poi scoprire che anche i carnefici sono vittime. Vittime del sistema del potere dei soldi e di chi ci governa, democrazie occidentali, teocrazie

\footnotetext{
${ }^{3}$ Martín Clavijo 2013: 178-179.
} 
medio orientali, capital comunismo orientale.

I pochi che manovrano $\mathrm{i}$ tanti. E le guerre sempre e solo per gli interessi di pochi mascherate da ideali, in cui i poveri fessi ci cascano e vengono indottrinati.

Consultando a varie riprese quel libro che le stava davanti pieno di sottolineature a matita, l'autrice ebbe modo di conoscere più approfonditamente questo dio, figlio di Zeus e di Era, e poi amante di Afrodite, da cui ebbe tra gli altri figli Eros e Armonia. Inoltre venne a conoscenza dei suoi guerrieri che non conoscevano nulla al di fuori della guerra, un po' come quelli che la stessa drammaturga definisce "diseredati americani" che si arruolano nei marines e lì vengono sottoposti, come evidenzia tra gli altri il richiamo ad un film del 1987, Full metal Jacket ${ }^{4}$ di Stanley Kubrick, al più accurato lavaggio del cervello, per diventare perfette macchine da guerra. Oltre a questa pellicola ne viene ricordata un'altra, Hiroshima, mon amour, di Alain Resnais, su soggetto e sceneggiatura di Marguerite Duras, uscita nel 1959. Inoltre due brevi battute :

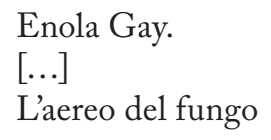

rinviano rispettivamente ai nomi del bombardiere che, alla guida del pilota Paul Tibbets, sganciò la prima bomba atomica su Hiroshima il 6 agosto 1945 e a quello da cui tre giorni dopo fu gettato un ordigno nucleare su Nagasaki, ponendo fine alla guerra col Giappone, ma provocando un numero elevatissimo di vittime, oscillante fra le cento e le duecento mila.

Immedesimandosi in Ares, per farlo parlare, questi del tutto inopinatamente divenne simpatico all'autrice, perlomeno per la ragione che, essendogli stata imposta dagli altri dei dell'Olimpo la guerra, non gli sarebbe stato possibile sfuggire al proprio tragico destino. Inoltre la Monaco, per così dire, aveva dato occasione al dio di fermarsi, fra un massacro e l'altro, concedendogli la possibilità di riflettere. In un'altra circostanza parlando di questo testo la drammaturga, mescolando ricordi della stesura primitiva e di quella successiva, mi ha detto:

Ares, la penultima verità, sono le riflessioni del dio greco e le sue reincarnazioni attraverso i secoli, focalizzandosi sul secolo appena trascorso: il pilota

${ }^{4}$ La Monaco, oltre ai libri, fa sovente riferimento nei suoi testi ai film. Ricordo che questo è ispirato al romanzo Nato per uccidere, dell'ex marine Gustav Hasford, esplicitamente citato nel testo della drammaturga. La pellicola, fortemente critica sulla guerra del Vietnam, uscì ad un anno di distanza da Platoon di Oliver Stone. 
americano che sganciò la bomba su Hiroshima, un marine in Vietnam (Born to kill, nato per uccidere), un soldato italiano in Bosnia colpito da tumore a causa dell'uranio impoverito, tecnici militari addetti ai bombardamenti tecnologici e intelligenti.

Va segnalato che il monologo per attore maschile era il primo scritto da Patrizia Monaco. In effetti la drammaturga, come farà altre volte, mostra di prediligere il ritmo mosso dei dialoghi. I pochi monologhi da lei ideati sino ad allora erano stati esclusivamente per voce femminile, in cui le era maggiormente semplice identificarsi. A quanto lei stessa osserva, la costruzione del pezzo la affascinò, perché "entrare in panni che aborrivo costituiva una sfida che mi consentì di attingere a risorse che non mi riconoscevo".

Ares, figlio di Zeus e di Era, schivato e deriso dagli altri dei per il carattere aspro e violento, viene scelto dalla Monaco per la spinta paradossale e ironica di fare quasi una difesa di ufficio di un dio terribile e litigioso, anche se in fondo sincero, contrapposto come è all'ipocrisia che caratterizza da sempre l'essere umano. Scavando nella "biografia" di Ares la drammaturga aveva evidenziato da un lato i tratti essenziali della nascita di un dio a cui è impossibile opporsi al proprio destino, e dall'altro la constatazione che egli si duole di essere il protettore di uomini prepotenti e brutali. Significativamente nella prima stesura invocava i soli Eros e Armonia, nati dalla relazione con Venere, mentre con lei aveva generato anche altri figli che verranno ricordati nella seconda redazione del testo, rendendo così quel dio una figura più inquietante: "Dicevano che io andavo in battaglia accompagnato dalla mia altrettanto antipatica prole Deimos e Fobos, la paura, il terrore. Con noi c'era anche [...] Eris, la Discordia"s.

Traendo spunto proprio dalle vicende del dio della guerra, l'autrice costruisce in pochi giorni uno svelto e denso monologo in cui la divinità classica si sdoppia in diversi personaggi, che, attraversando i secoli, rappresentano l'istinto di aggressività insita nell'essere umano, perennemente diviso tra la condanna della guerra e la sua esaltazione. In effetti lungo la storia gli uomini si sono rivelati non di rado inconsapevoli pedine nel gioco dei potenti. Nel contempo il personaggio di Ares assume anche la parte del guerrigliero, che scartate altre strade, decide di scegliere la via della violenza, in nome paradossalmente proprio di Eros e dell'Armonia. Già in questa redazione asciutta, secca e stringata il linguaggio di Ares è duro, crudo, aspro e lancinante. Nel lavoro la Monaco manifestava in maniera palese il forte disgusto verso chi vuole le guerre, svelando che queste sono dichiarate solo all'apparenza per motivi ideologici, mentre nella realtà vengono combattute per esclusive e bieche motivazioni economiche. Ares, che è volta a volta guerrigliero palestinese, marine USA e sé stesso, incontra ad un certo punto anche il pilota

\footnotetext{
${ }^{5}$ Monaco 2008: 207.
} 
che, avendo sganciato la bomba su Hiroshima, dovrebbe provare un minimo senso di colpa. La regista che allestì il componimento per la prima volta pigiò molto sul pedale della provocazione facendo indossare al protagonista una T-shirt con la foto delle Torri Gemelle crollate a New York soltanto due mesi prima. L'esecuzione dell'attore che lo interpretò sottolineò poi in maniera molto convincente ora $\mathrm{i}$ momenti tragici e ora quelli poetici che caratterizzavano la pièce, evidenziando come questa fosse un deciso manifesto pacifista. Il simbolismo poi che l'autrice, ispirandosi al teatro Noh giapponese, inserì in alcune didascalie in cui si parla di "tre rotoli di seta, uno rosso, uno verde e uno azzurro", posti in un angolo del palco, indicano con semplicità ma insieme efficacia il sangue versato nel corso dei conflitti armati, la giungla vietnamita in cui combatterono dai primi anni Sessanta del Novecento all'aprile 1975 i soldati del Vietnam e degli Stati Uniti e l'acqua del fiume Lete in grado di far dimenticare, bevendola, le ignominie commesse dagli uomini, verrà mantenuto anche nelle successive rappresentazioni per l'efficacia e la suggestione della resa visiva. In effetti il contrasto fra recitazione muscolare e riferimenti mitologici valeva a restituire appieno allo spettatore la carica eversiva che percorre e sostanzia il testo. Appesi oppure vicini ad un attaccapanni, collocato ai lati della scena, si notano alcuni oggetti dall'aspetto inquietante e minaccioso che costituiscono un insieme di notevole efficacia scenica: "una maschera antiga, un passamontagna nero, una borsa da computer, contenente un portatile, un elmo greco" e "un paio di scarponi anfibi neri, con dentro infilate delle calze dello stesso colore". Per tali motivi il copione in questa originaria versione verrà scelto per altre rappresentazioni date negli anni successivi che allora mise in scena il in eventi rilevanti, quali la marcia contro la dichiarazione della Guerra in Iraq nel 2003 e altre manifestazioni civili contro ogni forma di prevaricazione.

Un'attrice sensibile, Patrizia La Fonte, con cui allora la Monaco collaborava e che faceva parte dell'organizzazione copione, apprezzò la crudezza del linguaggio impiegato. Qualche giorno dopo la stessa attrice osservò in una e-mail:

ho trovato Ares bellissimo, forte, urlante, epico. Come è la guerra, che secondo me, giustamente, hai mostrato in tutta la sua potenza sanguinaria e in tutta la sua terribile ragion d'essere.

A me piace moltissimo. Non so che effetto farà alle direttrici della manifestazione romana spero che non la stiano a menare troppo col politically correct che a volte le distoglie dai veri temi del mondo.

Poco prima che un'altra regista, Francesca Covatta $^{6}$, le chiedesse sul finire dell'anno successivo di continuare il lavoro con altre figure mitiche, la Monaco,

\footnotetext{
${ }^{6}$ La richiesta della regista era dettata dalla volontà di inserire il nuovo testo in una rassegna teatrale intitolata Condominio Mitologico, che avrebbe dovuto essere realizzata qualche mese dopo ma che nella realtà non si concretizzerà.
} 
sconvolta dai fatti avvenuti a Genova durante il G8 svoltosi nel capoluogo ligure, aveva scritto Icaro 2001 che si apriva con un'indicazione illuminante, peraltro espunta nell'edizione a stampa edita qualche anno dopo perché ritenuta pleonastica:

ICARO 2001 è un ragazzo che fu alle manifestazioni no global del 17 marzo 2001 a Napoli e il 20 luglio dello stesso anno a Genova vide concludersi la sua breve vita. Nessun intento biografico nella storia di Icaro 2001, poiché vuole simboleggiare tutti i ragazzi con degli ideali e pertanto incarna il mito del giovane che volò verso il sole.

Va detto che quel copione è espressione diretta dello choc del G8, per le violenze consumate, culminate con l'uccisione di un ragazzo e le torture alla Scuola Diaz di Bolzaneto, e contrassegnate dall'impunità della polizia e dalle menzogne sui black block. Come molti, aveva notizie di prima mano da amici genovesi con cui faceva teatro e che erano rimasti sul posto. La Monaco vide tutto in televisione, anche se non fu veritiero come il documentario che uscirà anni dopo. Vedendo scorrere le immagini dell'evento sul piccolo schermo la drammaturga restò

colpita dall'assurdità di tutta la faccenda. Si riunivano i grandi della terra e la zona doveva essere limitata, gli abitanti dei vicoli ingabbiati e furono ordinate non so quante bare in anticipo. Non è una leggenda metropolitana, ma notizia di chi allora era in Prefettura.

Non impiegarono le forze di polizia locali ma un reparto di celerini provenienti da Roma.

Io sessantottina ancora nell'anima mi sentii fremere a quel nome e a quel che accadde.

A proposito di questo testo, che allora non venne rappresentato assieme ad Ares, la regista commentò a caldo:

Icaro è splendido, commovente. Davvero! Ho veramente pianto mentre lo leggevo. Mi spiace solo una cosa nella scelta di farne Carlo Giuliani cioè il fatto che essendo già morto racconti tutto al passato, mi sarebbe piaciuto riuscire $\mathrm{a}$ mettere in scena più al presente l'orrore e il terrore negli occhi di quei giovani.

Nel testo si alternano in modo funzionale le riflessioni dell'esanime Carlo Giuliani, già steso a terra, su quanto è accaduto in quei giorni, e la narrazione della vicenda fatta da Icaro e da suo padre Dedalo. Il monologo recitato da un Icaro seminudo, sdraiato su un tappeto di piume insanguinate, a indicare la sua avvenuta morte, narra in maniera dolente non solo i sogni e l'avventatezza dei giovani, ma anche la fatalità e l'infamia dei potenti. 
Il fatto che nel testo l'autrice non prendesse parte apertamente per il ragazzo morto, la cui figura non usciva esaltata, aggiunta alla non stigmatizzazione del giovane carabiniere che gli sparò, fece sì che la famiglia, in particolare, la madre di Carlo Giuliani, si opponesse recisamente alla messinscena dello spettacolo a Genova, che pure veniva richiesta da molti, come risultava da una plebiscitaria votazione in rete nel 2003. Il fatto è che secondo la Monaco il mito classico più consono per raccontare quel tragico evento era quello di Icaro. Per prima cosa l'autrice e la regista videro sulla scena, come si legge nella didascalia iniziale, "delle piume macchiate di un qualcosa che pare sangue". Alcuni "brani del monologo- annota la Monaco- scritti in corsivo, sono "la storia di Icaro". Altri invece, riportati "in piana esposizione contemporanea", che costituivano "il nucleo più ampio", erano scritti "in un linguaggio con cesure poetiche echeggianti, talvolta, assonanze e rime interne" ed era impreziosito dal rinvio a tre versi danteschi. Esemplare è a tale proposito la battuta iniziale di Icaro:

Per me si va nella città dolente.

Per me si va nell'eterno dolore.

Per me si va tra la perduta gente.

Il centro storico era diventato una gabbia

la città intera un labirinto fra i container

zona rossa da sorvegliare a vista

perché tanta paura quando chi ci governa ci riunisce?

$\mathrm{E}$ il ronzio incessante degli elicotteri.

Due ore stetti riverso

con gli occhi al cielo

occhi che qualcuno pietosamente

mi chiuse

ma io, ai piedi di quella chiesa

vidi e sentii tutto

anche attraverso le palpebre chiuse

il cuore aveva cessato di battere

ma non di soffrire

soffrivo per quel che vedevo e sentivo

quel che accadeva anche lontano da me

lontano da quella piazza grigia per i lacrimogeni

nelle belle e restaurate sale del Palazzo Ducale.

new economy

wto

new economy?

Del resto, prosegue la Monaco, "Il linguaggio impiegato nel copione non è

\footnotetext{
${ }^{7}$ Monaco 2008: 211.
} 
mimetico, come non è realistica la situazione drammatizzata: chi è morto parla trascendendo ogni verisimiglianza". Il linguaggio è da ballata, con qualche rima interna, citazioni da slogan di quel momento, quelli dei punk bestia -"produci, consumi e crepa"- e da altri letti sui muri di Genova.

L'autrice aveva inteso attribuire gli ideali del Maggio francese (che erano stati i suoi) a quel ragazzo.

Voglio credere - mi ha detto pochi giorni fa- fermamente che anche questi ragazzi di oggi abbiano degli ideali. Che si ribellino alle ipocrisie dei governi, tutti, senza distinzione, che pur di fare affari sacrificano le nostre vite come pedine di un gioco di cui solo loro pare sappiano le regole.

Poi, con un sorriso amaro, aggiunge commossa:

Ultimo Icaro, dalle ali di cera, forse con ideali più solidi di quelli espressi in Icaro 2001, Icaro 2016, Giulio Regeni, torturato a morte da un regime sostenuto e coccolato dai governi occidentali perché buon partner economico.

Infine, riprendendo la parte finale della battuta conclusiva di Icaro verso la conclusione del primo tempo di Condominio mitologico, l'autrice scrive:

Volate alto finché potete, per conquistare il futuro bisogna prima sognarlo.

L'importante non è di avere tante

idee. È di viverne una ${ }^{8}$.

La costruzione del monologo non è più come era in partenza ad una sola voce, poiché al resoconto del ragazzo morto riverso sull'asfalto, fa da contrappunto un'altra voce, fuori scena, che legge una versione del volo e della morte di Icaro nel mare Egeo. Lo spettacolo ha come base musicale il rumore degli elicotteri e delle ambulanze, capaci di dare un senso di minaccia incombente e da dittatura sudamericana.

Quasi subito nella Monaco matura il convincimento che il testo avesse le potenzialità per essere inserito, con opportuni ampliamenti e ritocchi, in un organismo più complesso, compatto e organico. Di qui la decisione di utilizzare nel prosieguo del lavoro una struttura non più lineare, come era avvenuto nella precedente stesura, ma ondivaga e maggiormente mossa. In effetti nella nuova riscrittura a cui lavorò con lena si incomincia quasi dalla fine della vicenda drammatizzata nel primo copione, vale a dire dall'Intifada palestinese, poi, per rievocare avvenimenti accaduti in diversi tempi, si va indietro, poi avanti

\footnotetext{
${ }^{8}$ Monaco 2008: 216.
} 
e ancora nuovamente indietro fino ad arrivare ai primordi e alle disavventure amorose di Ares e ai suoi rimpianti. In questo modo il dramma si carica di risonanze che, dilatandosi nello spazio e nel tempo, lo rendono più incisivo. $\mathrm{A}$ fare da contrappunto alle varie sequenze in cui si articola quella che era diventata la scena iniziale del lavoro è "il suono di un clarinetto basso". In questa parte per un verso vengono stigmatizzati gli interessi comuni per le guerre, cause di "sangue, fragore, sudore e morte per l'umanità di "fabbricanti di armi" e di "militari di carriera e per l'altro vengono difesi in maniera vibrante e commossa "tutti i disgraziati, gli sfruttati, i diseredati" che delle guerre sono le vittime incolpevoli. Nel contempo però vengono denunciati i danni gravissimi perpetrati dall'uomo ai danni del pianeta per i disboscamenti selvaggi dovuti all'impiego indiscriminato e criminale della chimica.

In particolare la Monaco, avvertita la necessità che per rendere compatto il testo occorreva intervenire con aggiunte, ripuliture testuali e qualche sottrazione, nonché con un lavoro attento di rimodulazione di quanto aveva scritto per assicurargli un più fluido collegamento, si mise prestissimo all'opera. Oltre ad assemblare i due primi testi, collocati non più uno di seguito all'altro, ne aggiunse un terzo, la cui stesura le venne suggerita dall'idea del prologo recitato da una Parca, tolto, lo accennavo in precedenza, alla vigilia del debutto del primo lavoro per non appesantirlo e snaturarlo. L'innesto di questa parte, oltre ad irrobustire il copione, arricchendolo e rendendolo maggiormente conforme ad un testo più dinamico ed articolato, contribuì non solo a dare a quel personaggio maggiore rilievo e spessore, ma anche a metterlo in stretto rapporto con altri creati proprio allora appositamente. Nella nuova struttura il copione ora era preceduto da un nuovo prologo, più lungo rispetto al precedente, detto dalla Parca Atropo, vale a dire da colei che ha il compito di recidere il filo della vita: "Eravamo tre, una iniziava la nuova spola, l'altra tesseva e io tagliavo".

Anche questa figura mitologica nella riscrittura subisce un cambiamento significativo, assommando all'originaria funzione di Parca quelle di "portinaia" del condominio e di "dottoressa specializzata in genetica".

L'ultima parte elaborata, come accennavo in precedenza, sul finire del 2002, venne inserita nell'unica scena del secondo tempo. L'argomento è, come dicono prima Giove e poi il dio Mercurio, le vicende di due divinità minori romane, quella dell'eloquenza, Aius Locutius, e del silenzio, Tacita Muta ${ }^{10}$. Il nome originario della giovane era Lara, figlia del fiumiciattolo Aimone, che confluisce nel Tevere. Alla giovane, come viene ricordato dapprima da Giove e poi da Mercurio tramutatosi a vista nel padre degli dei, è usata una violenza carnale, da cui

\footnotetext{
${ }^{9}$ Monaco 2008: 199.
}

${ }^{10}$ Del primo parlano tra gli altri lo storico latino Tito Livio ne La fondazione di Roma, 5. 32 e Cicerone ne La divinazione, 1. 101; della seconda Ovidio ne I fasti, 2. 615-616. 
nasceranno i Lari Compitali. Lo stupro della ninfa è preceduto dal taglio della lingua della giovane da parte di Giove per punirla di avere rivelato alla sorella le mire che questi nutriva su di lei. Lo spunto per costruire questa parte conclusiva del pezzo, più che essere ricavata dalla ristampa di un libro molto fortunato di Eva Cantarella ${ }^{11}$, era derivato dallo sgomento provocato nella drammaturga e in moltissime persone da due terribili esempi di orrore avvenuti proprio sul finire del 2002 (la guerra in Cecenia con le repressioni di Vladimir Putin e un fatto efferato di cronaca nera italiana: lo stupro e la successiva mutilazione della giovanissima Desirée Piovanelli, uccisa il 28 settembre quasi per gioco da un gruppo di coetanei, su istigazione di un adulto). Di certo questa sequenza, scandita da scontati slogan misogini, da luoghi comuni razzisti e da dichiarazioni improntate dal più scontato e bieco machismo, nonché da una battuta meta teatrale pronunciata da Mercurio ("Non hai il senso del teatro: a teatro non si racconta, tutto avviene qui e ora" ${ }^{12}$ ) aggiunge maggiore equilibrio e compattezza alla pièce, caricandola di valenze molto incisive.

L'articolazione del lavoro, capace di saldare felicemente le tre parti di cui si era venuta costituendo, prevedeva ora tre parti svelte, veloci e compatte.

La mancata realizzazione del progetto della Covatta convinse la drammaturga a mettere per il momento da parte la nuova versione del testo, limitandosi a proporre i due asciutti e stringati monologhi al pubblico separatamente. Già in queste redazioni il testo faceva parte a pieno titolo dei lavori della drammaturga a contenuto spiccatamente civile e sociale, che caratterizzano una parte significativa della produzione dell'autrice italiana.

Il titolo della pièce, che le era stato suggerito dalla regista sopra ricordata, venne però mantenuto, in quanto si confaceva bene al nuovo copione, imperniato su un tema mitologico capace di suscitare reazioni indignate nel pubblico. In effetti gli argomenti mitologici utilizzati dalla drammaturga italiana non sono mai di natura libresca, in quanto costituiscono materiale che sa rievocare con forza episodi che provocano brividi e sgomenti profondi. Analizzando con maggiore attenzione il testo, attraverso il grido di vibrante condanna contro le guerre e ogni forma di prevaricazione nei confronti di donne e uomini, condannati ad un'esistenza di miseria e di atroci sofferenze, si coglie la volontà dell'autrice italiana di innescare un risveglio di coscienza civile in un pubblico che stava intorpidendosi. Significativamente l'Epilogo recitato dalla Parca, nell'atto di mostrare la maschera di Lara a cui è stata strappata la lingua, inizia con tono tutt'altro che melodrammatico, a dispetto di quanto lei stessa esclama in una battuta meta-teatrale:

${ }^{11}$ Cantarella 1985. Il libro della Cantarella venne ristampato nel 1996, nel 1998, nel 2001, nel 2003, nel 2006, nel 2008 e nel 2015. La Cantarella pubblicherà nel 2015 un altro volume, Passato prossimo: donne romane da Tacita a Sulpicia.

12 Monaco 2008: 219. 
Gridate! Gridate la vostra rabbia perché è l'unica cosa che non possono toglierci, e la rabbia di una donna sfruttata, mutilata e stuprata salirà fino al cielo $\mathrm{e}$ scenderà nelle viscere dell'inferno, risveglierà demoni e angeli e arriverà un giorno persino fino agli uomini. Quel giorno la nostra parola, anche se non è d'oro come quella degli uomini, avrà il suo peso, anche se fatta di piume e di vento, d'aria o di fuoco.

Troppo abbiamo sopportato, abbiamo sopportato più di quel che è umano sopportare, neppure le bestie hanno sofferto come noi. Le bestie, anche le tigri mangiatrici di uomini, che adesso proteggono come categorie in via di estinzione $^{13}$.

Avendo saputo che nel 2007 era stata bandita la terza edizione del Premio Fersen per la promozione e la diffusione della drammaturgia contemporanea italiana, la Monaco decise di parteciparvi recuperando la redazione del lavoro, accantonata in attesa di tempi migliori. L'autrice si limitò questa volta ad apportare qua e là nuovi piccoli ritocchi, come l'espunzione originaria dei sottotitoli dei due primi lavori e l'inserimento di qualche didascalia più ricca e robusta, ma soprattutto la migliorò con una scansione più solida ed equilibrata.

Tutti gli episodi del copione, che verrà edito a stampa nel 2008 assieme a tutti i testi vincitori dei quell'edizione del Fersen, si svolgono all'interno di un singolare condominio. Tecnicamente però la struttura del lavoro aveva virato dal monologo al dialogo, decisamente più efficace per raccontare episodi che non avrebbero potuto essere messi in scena secondo i canoni del dialogato tradizionale. La originaria stesura dell'opera mostrava e non rappresentava con efficacia i fatti drammatizzati, come avviene in quella attuale. Interessante è ciò che afferma l'autrice,

l'attore interpreta più personaggi come in molti monologhi, ma in questo caso il gioco teatrale si fa più scoperto: i personaggi sono presentati al pubblico senza il filtro della rappresentazione.

Nel primo tempo, suddiviso in due scene, dopo la sequenza iniziale affidata al solo Ares, segue una scena che vede l'alternanza di battute di Icaro e di commenti della Parca, collocati con una sapiente tecnica cinematografica. A quest'ultima figura mitologica all'inizio dice una battuta rivolta al pubblico nella quale viene denunciata la scarsa attenzione dei governi di tutti gli Stati per curare chi è affetto da malattie:

Studio le persone colpite da malattie devastanti e so con certezza cosa capiterà ai loro figli e nipoti: Se potessi trovare una cura, se i governi mi dessero almeno

\footnotetext{
${ }^{13}$ Monaco 2008: 221-222.
} 
una parte di quello che è destinato alle guerre allora non starei a tormentarmi sulla forza della conoscenza e l'inutilità della stessa ${ }^{14}$.

Come già era accaduto in un suo testo del 2000, La strada verso il cielo, la battuta riflette una situazione personale vissuta dall'autrice: la necessità di cure per un tumore maligno, dalla prognosi infausta, che per fortuna si è risolto positivamente.

Il secondo tempo invece, costituito da un'unica scena, è preceduto e concluso dalla Parca. Immediatamente dopo compaiono nell'ordine Mercurio e la Parca, Giove e la ninfa Lara e poi a seguire nuovamente Giove e Mercurio. Inframmezzato tra queste due sequenze vi è l'accenno allo stupro compiuto nei confronti della giovane fanciulla, che diventerà col nuovo nome di Tacita Muta, la madre di due gemelli.

Interessante è la lettura della breve scheda sul primo testo dovuta alla penna dalla stessa drammaturga dopo la sua riscrittura:

ARES, figlio di Zeus e di Era, era il Dio della guerra. Gli altri dei, compreso il padre Zeus, lo schivano e deridono per il suo carattere litigioso e turbolento. Anche agli Ateniesi, uomini colti e dediti alla guerra solo in caso di necessità, era inviso. Scendeva in guerra senza parteggiare per nessuno, accompagnato dalla sorella Eris (Discordia) e dai figli Deimos (Terrore) e Fobos (Paura). Questi ultimi li ebbe da Afrodite, la sua amante preferita, che gli generò anche una prole più simpatica: Eros e Armonia.

Subito dopo la Monaco aggiunge: "In nome dell'Amore e dell'Armonia, paradossale, ripetitivo e lancinante è il linguaggio del dio dal membro eretto, che ci conduce alla penultima verità, quella del mito".

Durante un incontro l'autrice mi ha confidato che la riscrittura l'aveva soddisfatta appieno: "Io vedo con gli occhi della mente la scena dove si svolge quello che scrivo e quindi quel che vidi mi convinse che era la pièce giusta”.

Parlando alla regista della messinscena di Ares la Monaco annota:

ci ho pensato a lungo. La bomba atomica ha avuto la conseguenza della fine della seconda guerra mondiale, l'assetto dato dai vincitori dopo tale guerra ha portato a tutte le guerre seguenti, compresa questa. Per cui io ho lasciato il dialogo col pilota, che d'altro canto è un soldato che ha obbedito agli ordini, roso dal rimorso, non un martire della Jihad... (se poi è vero) quindi anche le battute dovrebbero essere cambiate radicalmente ed entreremmo nel territorio minato del terrorismo, che a me non fa paura, ma è che al momento non si sa nulla. Sapessimo di più e per certo... Ho preferito fare un aggiunta, al

${ }^{14}$ Monaco 2008: 199-200. 
colloquio con l'ombra, qualche riga sui quattro piloti degli attentati dell'undici settembre. Io preferisco raccontare così un atto terroristico avvolto ancora nel mistero, ma che si rifà di più ai kamikaze che ai piloti americani. Vedrai che se ci pensi bene è così. Ti ho parlato del poster a Catania: la bomba atomica da una parte e le due torri dall'altra. La bomba è stata un atto che molti giustificano, come adesso la ritorsione, quindi il passato può far riflettere sul presente.

Pertanto il nuovo testo era ora costituito non da due ma da tre parti, sapientemente fuse. In questa versione lo spettacolo risultava indubbiamente più efficace come resa scenica. Nella versione approntata per il concorso, che trova puntuale riscontro nella stesura comparsa a stampa l'anno successivo, la pièce appare costruita, afferma l'autrice, come una libreria dell'Ikea, e cioè in maniera modulare. A tale proposito la Monaco mi ha detto di recente:

Poiché mi piace paragonare il mio lavoro a quello di un artigiano, la parola inglese playwright infatti significa letteralmente costruttore di commedie, il primo ripiano nacque su sollecitazione di una regista, Maddalena Fallucchi per la rassegna Lo specchio di Narciso con una figura maschile fra mito e contemporaneità". Il titolo è Ares, o la penultima verità. L'aggettivo indica che la verità finale non ci è dato a noi umani conoscerla.

Nella versione definitiva la pièce è un testo che può rientrare nel filone del teatro di narrazione ${ }^{15}$. A interpretarlo sono sufficienti due soli attori, uno maschile e uno femminile, in quanto i personaggi non interagiscono fra loro se non in pochissimi momenti. Nelle altre sequenze invece si tratta di blocchi di monologhi contrapposti o di monologhi classici, preceduti da un prologo e conclusi da un epilogo, che hanno come unica voce la sopra ricordata Parca, portinaia e dottoressa. In questa nuova versione della pièce l'autrice, oltre a rivelare un grande abilità, dimostra la naturale attitudine a fondere teatro, sociale e analisi del presente.

Accennavo in precedenza che l'azione della pièce si svolge in un singolare condominio, la cui portinaia è una dottoressa specializzata in genetica ma che fu, in altri tempi, una Parca. Nel condominio, che ha la struttura della doppia elica, si intrecciano tre storie che presentano tra loro qualche inquietante assonanza. Gli inquilini sono alquanto bizzarri. Ares, lo accennavo in precedenza, è oltre che il dio greco della guerra, il pilota che sganciò la bomba su Hiroshima,

${ }^{15} \mathrm{Va}$ precisato che in questa definizione nessun autore si è riconosciuto, in quanto per riprendere Ascanio Celestini, uno degli esponenti più significativi del filone, "Questo teatro di narrazione in realtà non esiste come un genere unico $[. .$.$] perché non c'è una tradizione$ direttamente legata al teatro e non c'è perché il teatro di narrazione nasce spesso come percorso individuale. E questa credo sia una delle poche cose che hanno in comune quelli che fanno questo lavoro" (Celestini 2003: 21). 
un marine in Vietnam e un soldato italiano in Bosnia; Icaro è anche un ragazzo morto a Genova, la città della Monaco, nel luglio 2001, durante il G8; Tacita Muta, dea minore dei Romani, è la donna che viene stuprata un po' così, per passatempo o per noia.

I tre inquilini non si incontrano, solo la Dottoressa-Parca li conosce e commenta le loro vite e le loro azioni con la comprensione di una saggia portinaia che ha a cuore la sua comunità.

Al concorso a cui partecipò nel 2007, il copione risultò secondo classificato nella categoria Opera drammaturgica (due atti). Articolata in due tempi di diversa misura scritti a distanza di un anno l'un dall'altro, la pièce si legge ora tra le pp. 197- 222 del volume che raccoglie tutti i testi vincitori della terza edizione del Fersen (Roma, Editoria \& Spettacolo, 2008). La motivazione del riconoscimento del testo della Monaco, si legge alla pagina 11 del volume sopra ricordato:

L'autrice - di cui è noto l'impegno nel teatro al femminile - si cimenta nel genere socio-politico con scrittura esperta ed efficace, realizzando l'incontro fra il Mito e il nostro tempo. Il risultato è un testo grinçante (alla Anouilh), nel quale l'esperienza della Monaco evita eccessi didascalici.

Nella sesta delle dense pagine introduttive al volume il compianto critico Ugo Ronfani, osserva:

Rispondono alle intenzioni di affrontare problematiche attuali, direttamente o con riporti metaforici, altri testi prescelti, come Condominio mitologico, di Patrizia Monaco, dove il genere socio-politico viene trattato mediante l'incontro fra il Mito e il nostro tempo.

I tre personaggi non si incontrano mai. Forse -osserva con ironia la Monaco- non sono socievoli o forse hanno soltanto orari diversi. 


\section{Bibliografia}

Campbell, J. (2004), Il potere del mito. Parma: Guanda.

Cantarella, E. (1985), Tacita Muta: la donna nella città antica. Roma: Editori Riuniti.

Celestini, A. (2003), "Il vestito della festa: dalla fonte orale a una possibile drammaturgia", Prove di drammaturgia 2: 21.

Martín Clavijo, M. (2013), "El monólogo teatral en la dramaturgia italiana femenina de finales de siglo", in González de Sande, M. M. (ed.), Escritoras italianas desde el siglo XV hasta nuestros días. Madrid, Maia Ediciones: 178179.

Martín Clavijo, M. (2016), "L'altro volto della violenza", in Monaco, P., Donne in lotta. Roma, Aracne: 125-137.

Monaco, P. ( $\left.{ }^{2} 2008\right)$, Condominio mitologico, in Il Premio Fersen per la promozione e la diffusione della drammaturgia contemporanea italiana. Roma, Editoria \& Spettacolo: 197-222.

Monaco, P. (2016), Donne in lotta. Roma: Aracne.

Trovato, R. (2002), Parole e scene di un secolo in Liguria. Alessandria: Edizioni Dell'Orso.

Trovato, R. (2012), "La scrittura come vita e come gioco. Il teatro di Patrizia Monaco", in Martín Clavijo, M. et al. (ed.), Las voces de las Diosas. Sevilla, Arcibel: 1321-1345.

Trovato, R. (2014), "Dalla tela alla scena. Analisi drammaturgica di Sherazade va in Occidente di Patrizia Monaco", Revista Internacional de Culturas E Literaturas: 1-13.

Trovato, R. (2016), "La verità del personaggio", in Monaco, P., Donne in lotta. Roma, Aracne: 9-28. 